\title{
Full mouth rehabilitation of a patient with mutilated dentition
}

\author{
Priyanka Dhumal $^{1 *}$, Rajeev Singh ${ }^{2}$, Gaurang Mistry ${ }^{3}$, Asha Rathod ${ }^{4}$ \\ ${ }^{1}$ Post Graduate Student, ${ }^{2,4}$ Professor, ${ }^{3}$ Professor and Head, Dept. of Prosthodontics, D Y Patil University- School of Dentistry, Navi \\ Mumbai, Maharashtra, India
}

*Corresponding Author: Priyanka Dhumal

Email: priyankadhumal2692@gmail.com

\begin{abstract}
Excessive occlusal wear of dentition can result in pulpal injury, occlusal disharmony, impaired function and esthetic deformity. Wear of teeth can be in the form of attrition, abrasion, erosion, abfraction etc. Severe loss of anterior teeth results in loss of anterior guidance this compromise's the posterior teeth during excursive movement. Such excessive wear and abnormalities of teeth often impairs patients basic activities like mastication, speech and esthetics affecting patients life style. Therefore reconstruction of the mutilated dentition is necessary. Full mouth rehabilitation combines esthetics with the science of restorative dentistry to improve the health and function of the patient. This clinical case report describes the use of hobo twin-stage procedure for rehabilitation of a patient with severe tooth wear and reduced vertical dimension of occlusion.
\end{abstract}

Keywords: Hobo twin-stage, Mutilated, Splint, Myofacial pain dysfunction.

\section{Introduction}

Full mouth rehabilitation is achieving balance between esthetics and function and henceis a challenging procedure. This may get difficult if the restorative space required is limited as in the case of a mutilated dentition. Efforts should be made for meticulous treatment planning with a multidisciplinary approach for successful outcomes. The most critical aspect in successful full mouth rehabilitation is a combination of stable occlusion and orthopedically stable temporomandibular joints. Occlusal surface wear of the teeth in an individual is a gradual process during the lifetime. It leads to loss of vertical dimension in an individual with functional and esthetic discomfort in everday life. So the prosthetic treatment should be performed with extreme care to achieve a good prognosis for the patient. ${ }^{1}$

This clinical case report highlights the use of hobo twin-stage procedure for rehabilitation of patient with severe tooth wear and reduced vertical dimension of occlusion.

\section{Case Report}

A 48-year-old male reported to Department of Prosthodontics, D Y Patil University- School of Dentistry, Navi Mumbai, with a chief complaint of replacement of missing teeth and difficulty in chewing. The patient gave no significant medical history and no signs of temporomandibular joint disorder or myofacial pain dysfunction.

Patient gave a history of fixed partial denture in maxillary and mandibular anteriors which had fractured 8 years ago.

\section{Clinical Findings}

Extraoral examination revealed no facial asymmetry or muscle tenderness.

Intraoral examination revealed severe attrition and abrasion with 141636374647 missing in upper and lower arch. Draining abscess was seen in relation to 38.Drifting of maxillary and mandibular anterior teeth was seen due to open contacts. Porcelain fused to metal bridge on 252627 was present. 15 was supraerupted and 24 was rotated mesially (Fig. 1 and 2). Orthopantomogram (OPG) of patient further confirmed excessive wear with pulpal exposure in relation to maximum teeth present in the mouth. There was root resorption seen in 313241 . Periapical radiolucency was seen in relation to 38 (Fig. 3).

No gross abnormalities were noted in the overall soft tissues of the lips, cheeks, tongue, oral mucosa, and pharynx.

Approximately $3 \mathrm{~mm}$ of loss in Vertical Dimension was established. According to turner and Missirlian classification category 1 shows excessive wear with loss of vertical dimension. The closet speaking space is more than $1 \mathrm{~mm}$ and interocclusal space is more than $4 \mathrm{~mm}$ and has some of facial contour that includes drooping of the corners of mouth. Patient was a class 1 category according to Turner and Missirlian classification. Full mouth rehabilitation with restoration of $3 \mathrm{~mm}$ vertical dimension using hobo twin stage technique was planned to rehabilitate the worn out dentition in functional harmony while providing group function occlusion during eccentric movements. ${ }^{2}$

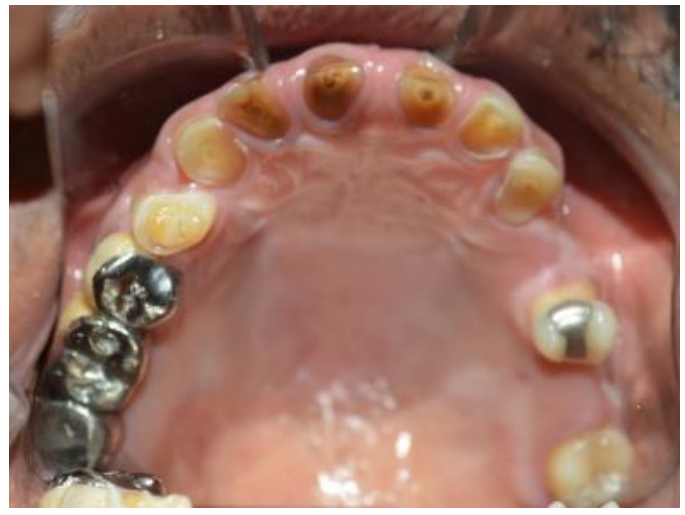

Fig. 1: Intraoral view of maxillary arch 


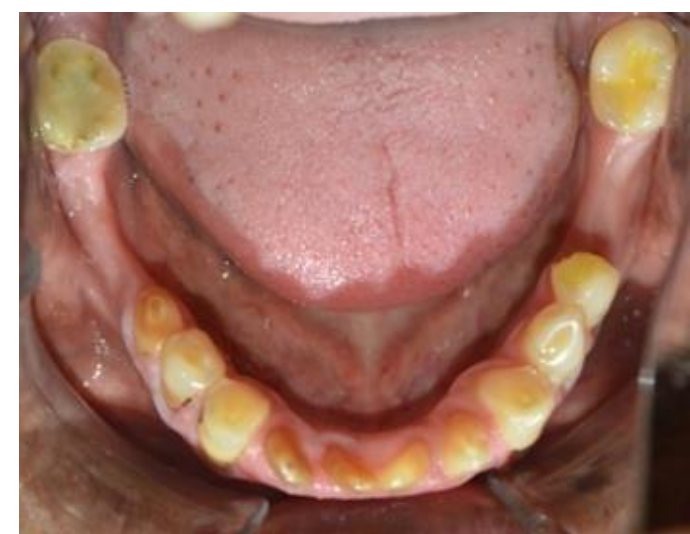

Fig. 2: Intraoral view of mandibular arch

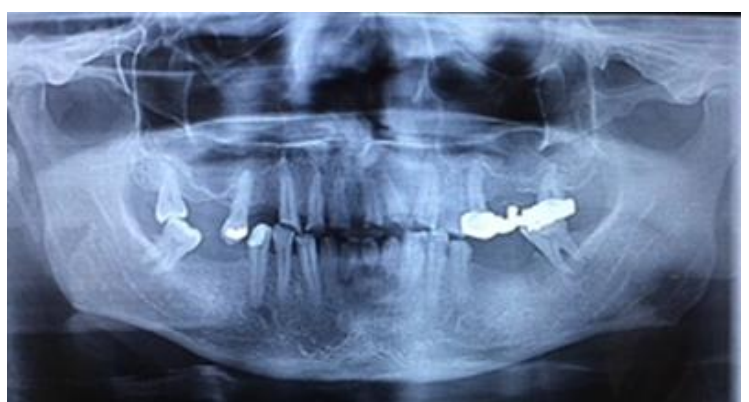

Fig. 3: Preoperative OPG view

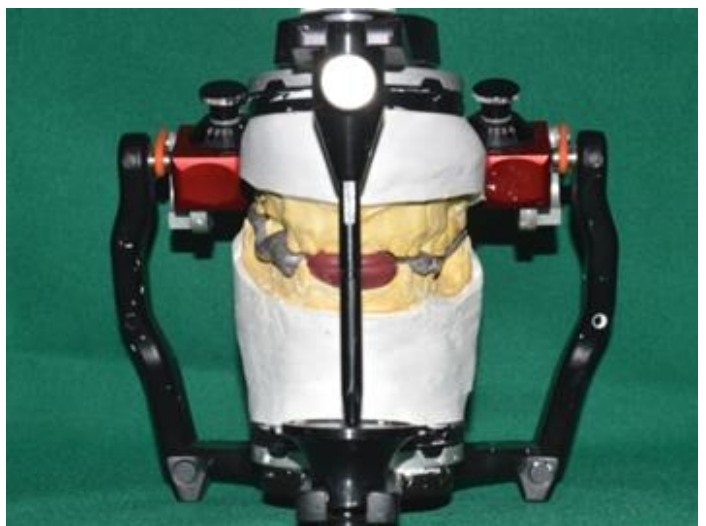

Fig. 4: Diagnostic mounting

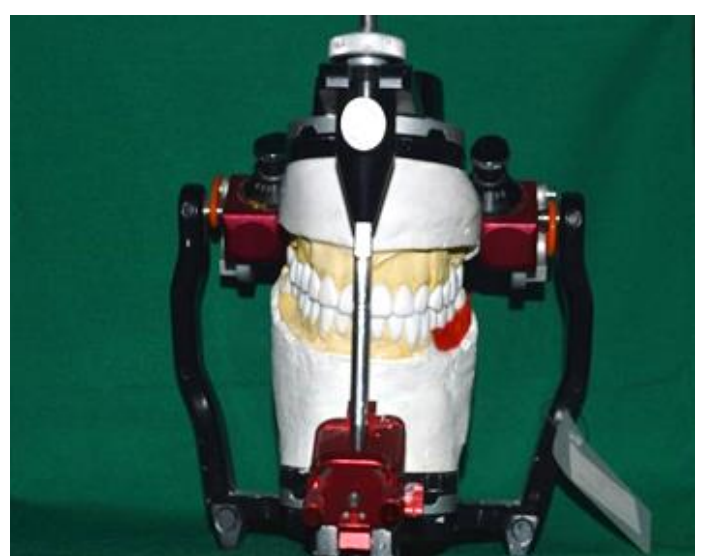

Fig. 5: Wax-up in centric

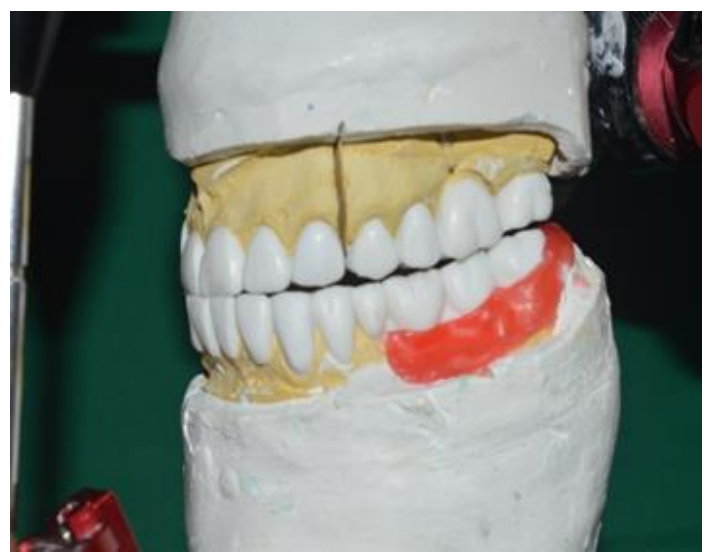

Fig. 6: Wax-up in protrusive

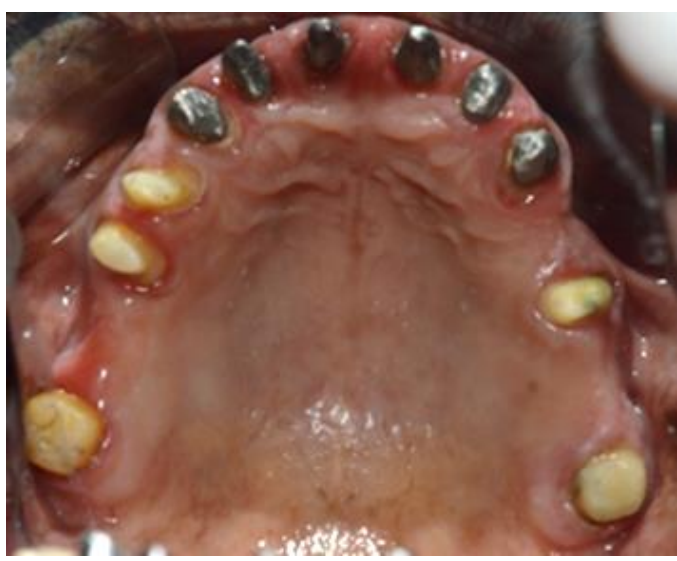

Fig. 7: Tooth preperation for maxillary arch

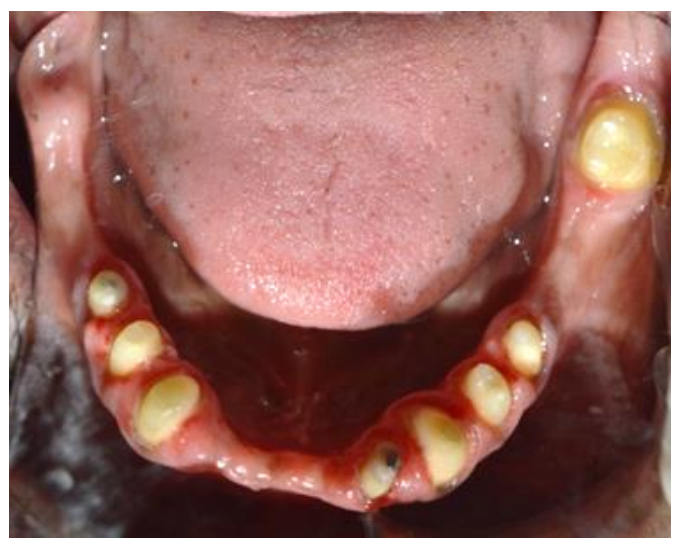

Fig. 8: Tooth preparation for mandibular arch

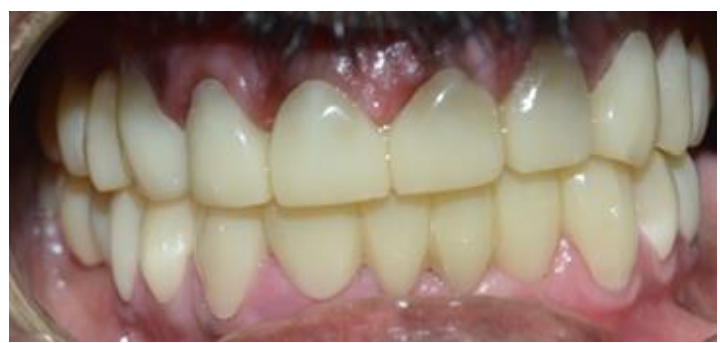

Fig. 9: Temporaries in centric occlusion 


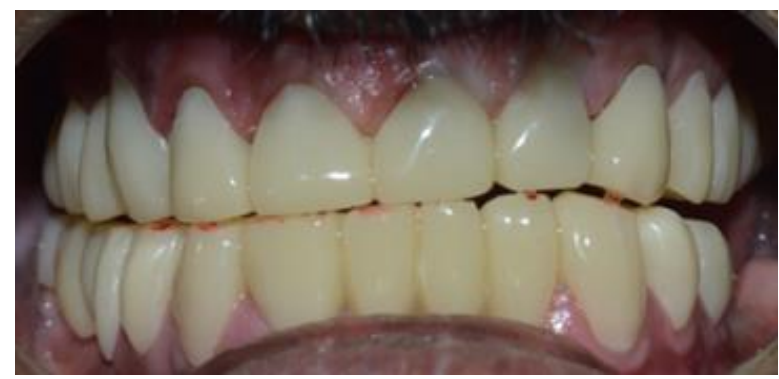

Fig. 10: Temporaries in protrusion with posterior disocclusion

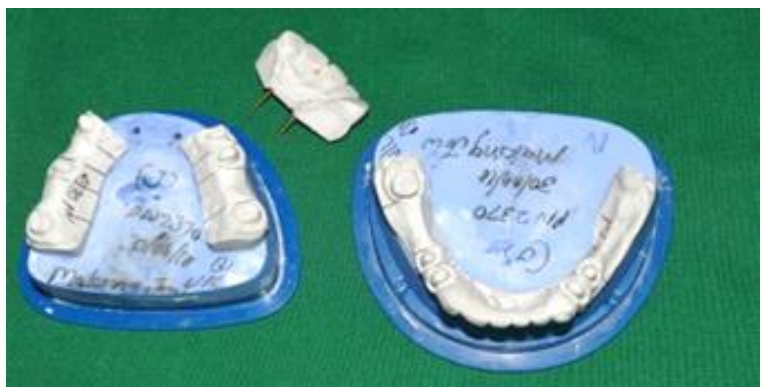

Fig. 11: Maxillary and Mandibular final models with maxillary anterior segment separated

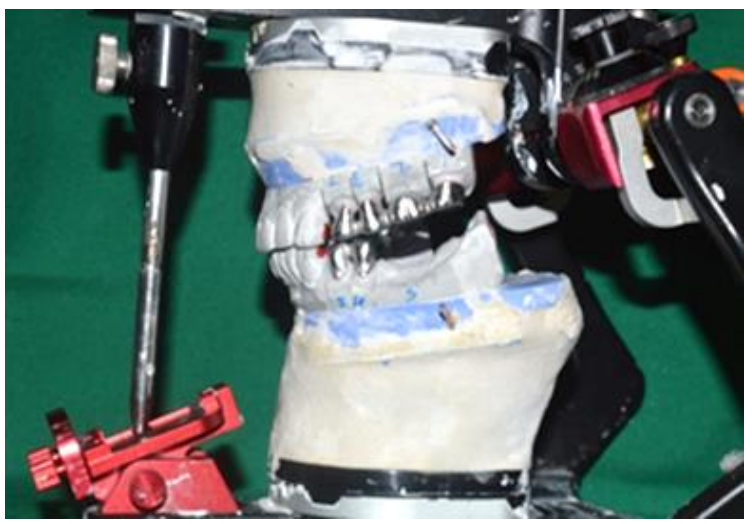

Fig. 12: Metal copings for upper and lower left side

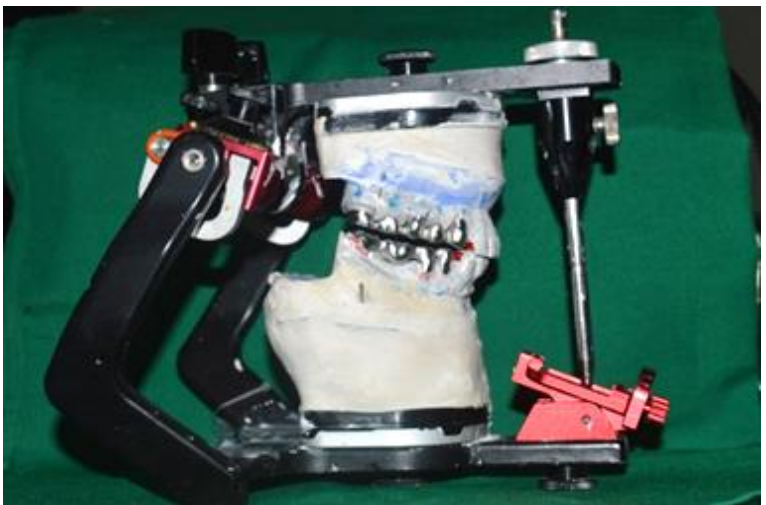

Fig. 13: Posterior metal copings for upper lower right side.

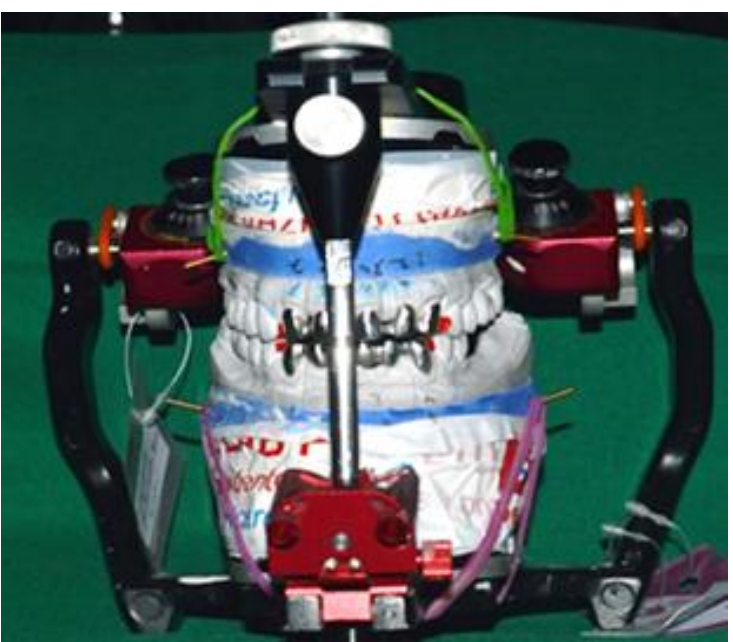

Fig. 14: Anterior metal coping for Maxillary and Mandibular arches

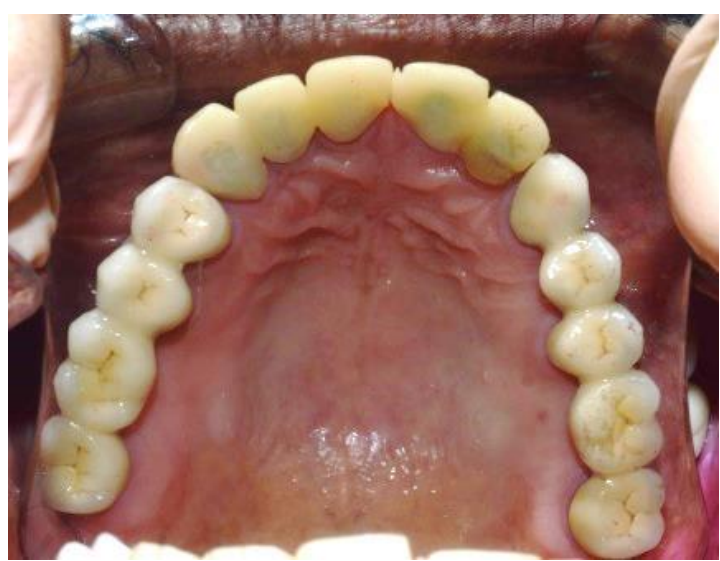

Fig. 15: Cementation of upper posteriors

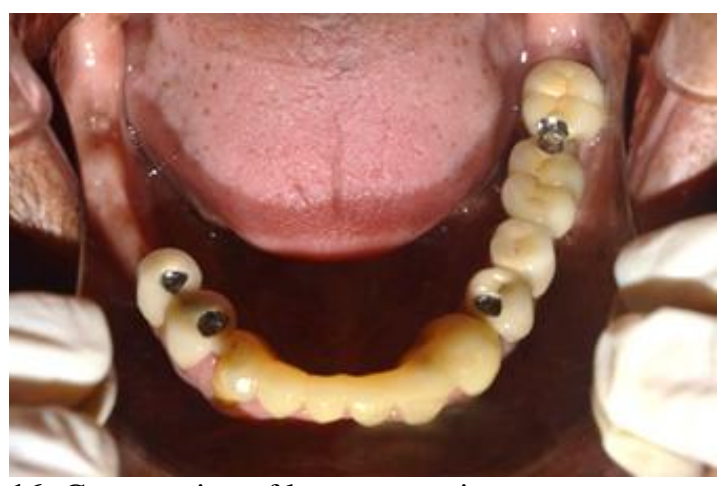

Fig. 16: Cementation of lower posteriors

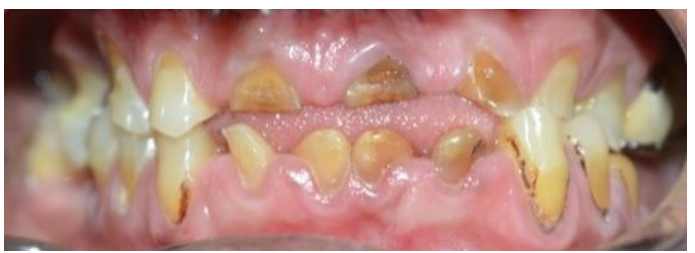

Fig. 17: Pre operative intraoral view 


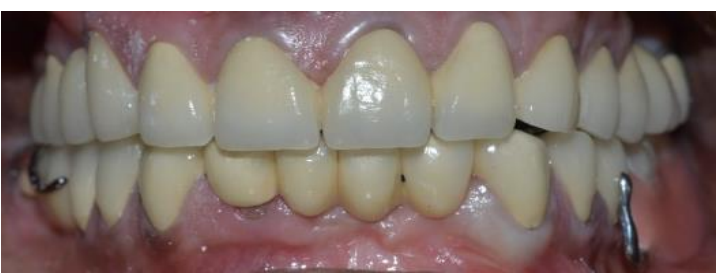

Fig. 18: Post operative intraoral view

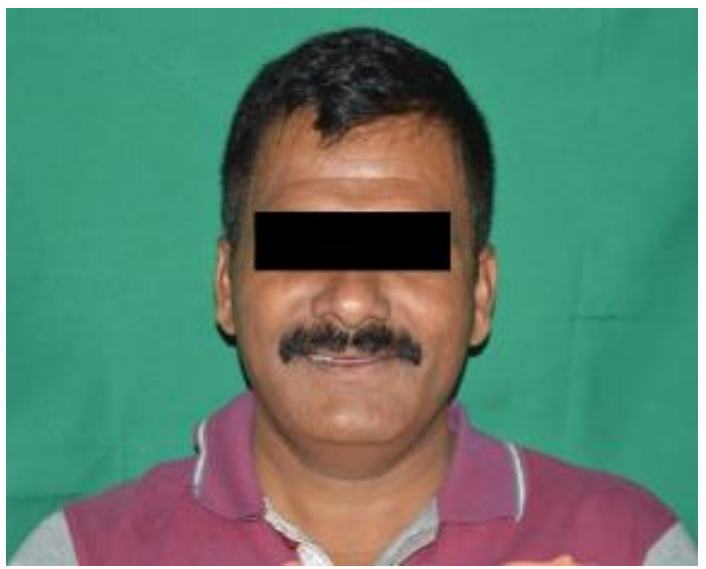

Fig. 19: Pre-operative extraoral picture

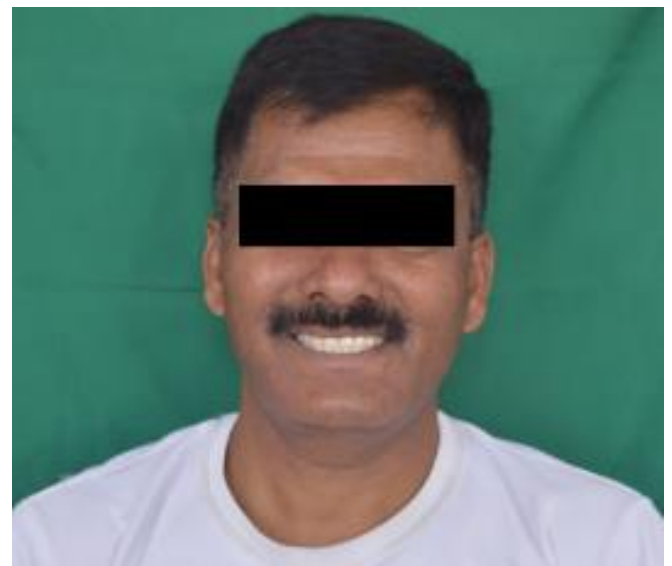

Fig. 20: Post-operative extraoral picture

\section{Procedure}

Maxillary and mandibular impressions were made in the irreversible hydrocolloid impression material and diagnostic casts were obtained. Based on patient's loss on vertical dimension, increase of $3 \mathrm{~mm}$ in the vertical dimension of occlusion was carried out using an anterior jig and interocclusal record based on the evaluation of the mounted diagnostic cast in centric relation. Then the maxillary cast was mounted using face-bow transfer onto a semi-adjustable articulator and the mandibular cast was mounted using the jig and centric relation record. ${ }^{3}$ Diagnostic wax-up was done according to hobo condition 1 with sagittal condylar path inclination $25^{\circ}$, bennett angle $15^{\circ}$, anterior sagittal inclination $25^{\circ}$, lateral wing angle $10^{\circ}$. Condition 2 values were sagittal condylar path inclination $40^{\circ}$, Bennett angle $15^{\circ}$, anterior sagittal inclination $45^{\circ}$, lateral wing angle $20^{\circ}$ at the established new vertical to see the final outcomes. ${ }^{4}$
Then an occlusal splint was provided to the patient as part of reversible treatment modality to evaluate adaptation of the patient to altered VDO. The patient was kept in observational period of 6 weeks before the definitive restorative phase of rehabilitation was started. ${ }^{5}$ The teeth were endodontically treated and cast post and core were done in 111213212223 order to receive stage I chair side temporaries. The definitive phase for metal-ceramic tooth preparations was completed for the entire dentition with minimal occlusal reduction; Stage II heat cure temporaries were fabricated using the putty index of the diagnostic waxup and cemented with $\mathrm{ZnO}$ non-eugenol cement. For three months, interim restorations were adjusted, and used as a guide for the definitive oral rehabilitation. ${ }^{5} \mathrm{~A}$ final full arch impression for maxillary/mandibular teeth was made using polyether impression material and casts were poured in die stone. This assembly was mounted on semi-adjustable articulator using the face-bow, Lucia jig, and centric record at the previously determined vertical dimension. Articulator was programmed to different condition values of Hobo's twin stage procedure. ${ }^{6}$ The metal copings were tried in and adjusted as needed. The definite restoration planned was DMLS crowns for better marginal fit of the prosthesis. Permanent cementation was done with GIC type I luting cement. Functional impression and altered cast was fabricated for cast partial denture in relation to 3637 for kennedy's class II. ${ }^{7}$

\section{Discussion}

In this case, the patient was carefully monitored for a month to evaluate the adaptation to the removable occlusal overlay splints. ${ }^{2}$ Patient had fixed partial denture which had dislodged that further lead to resorption of the exposed tooth structure. In addition, maxillary posterior base metal prostheses might have accelerated the wear of mandibular teeth and unbalance of wear rate. Minimally invasive prosthodontics was the key for rehabilitation of this dentition. ${ }^{8}$ Cast post and core were carried out in order to achieve favourable crown root ratio. Also the patient's adaptation to the provisional restoration was monitored for 2 months. There was no discomfort, wear, and muscle fatigue observed during that period. The restoration of VDO was determined by patient's physiologic factor like interocclusal rest space and speech. ${ }^{8}$

Group function occlusal scheme was planned, wherein the posterior cuspal morphology was contoured as per standard values formulated by the hobo's twin stage technique and anterior teeth caused disocclusion of posteriors in protrusion. ${ }^{3}$ The idea was to fulfill the requirements of an ideal occlusion, for a harmonious functioning of the stomatognathic system.

The idea for taking TMJ scan before and after temporization in this case was to study and evaluate the position of the condyles in fossa. ${ }^{9}$

There had to be modifications made in the treatment plan due to missing 14 . The posterior segment could not be separated from 13 and canine had to be included as the abutment for the bridge. 
The lower left side was edentulous and option for implants with fixed prosthesis was ruled out due to insufficient bone. Cast partial denture was prosthesis of choice as attachment on 35 was not possible due to compromised condition of the abutments.

The rehabilitation using restoration of anterior crowns and RPD providing posterior support is economical and common for many patients who require the treatment of teeth wear because of financial reasons or less bone. However the patients must be encouraged and properly educated to use the RPD, as failure to do so may lead to excessive occlusalload or supra-eruption of the maxillary teeth opposing RPD. Regular checkup for the occlusal adjustments CPD fitting is essential. ${ }^{10,11}$

A 6 months follow-up was done, there was no abnormalities seen in relation to the performed treatment.

\section{Conclusion}

The restoration of form, function, and esthetics in mutilated dentition is a demanding procedure and should be carried out only when absolutely indicated. Proper diagnosis and multidisciplinary treatment planning with adequate knowledge and judgement are paramount success. In this clinical report, restoring vertical dimension of occlusion using removable occlusal overlay splint and following minimally invasive procedure with fixed provisional based on accurate diagnosis showed successful full mouth rehabilitation for severely worn down dentition.

\section{Source of funding}

None.

\section{Conflict of interest}

None.

\section{References}

1. Schuyler $\mathrm{CH}$. The function and importance of incisal guidance in oral rehabilitation. J Prosthet Dent 1963;13:1011-29.

2. Johansson A, Johansson AK, Omar R, Carlsson GE. Rehabilitation of the worn dentition. J Oral Rehabil 2008;35:548-66.

3. Brown KE. Reconstruction considerations for severe dental attrition. J Prosthet Dent 1980;44:384-8.

4. Hoyle DE. Fabrication of a customized anterior guide table. $J$ Prosthet Dent 1982;48:490-1.

5. Dawson PE. Evaluation, diagnosis and treatment of occlusal problems. St Louis: C V Mosby Co. 1974. p. 148-9

6. Turner KA, Missirlian DM. Restoration of the extremely worn dentition. J Prosthet Dent 1984;52:467-74.

7. Hobo S. Twin-tables technique for occlusal rehabilitation: Part I-Mechanism of anterior guidance. J Prosthet Dent 1991;66:299-303.

8. Hemmings KW, Darbar UR, Vaughan S. Tooth wear treated with direct composite restorations at an increased vertical dimension: results at 30 months. J Prosthet Dent 2000;83:28793.

9. Sato S, Hotta TH, Pedrazzi V. Removable occlusal overlay splint in the management of tooth wear: a clinical report. $J$ Prosthet Dent 2000;83:392-5.

10. Jahangiri L, Jang S. Onlay partial denture technique for assessment of adequate occlusal vertical dimension: a clinical report. J Prosthet Dent 2002;87:1-4.

11. Prasad S, Kuracina J, Monaco EA Jr. Altering occlusal vertical dimension provisionally with base metal onlays: a clinical report. J Prosthet Dent 2008;100:338-42.

How to cite the article: Dhumal P, Singh R, Mistry G, Rathod A. Full mouth rehabilitation of a patient with mutilated dentition. J Dent Specialities 2019;7(2):111-5. 